\title{
THE NUMBER OF PHENOTYPES AMONG THE CONDITIONED LINES OF NICOTIANA RUSTICA
}

\author{
PHILOMENA TOWEY and J. L. JINKS \\ Department of Genetics, University of Birmingham, Birmingham B/5 2TT
}

Received 12.iv.76

\section{SUMmary}

\begin{abstract}
Analysis of the cumulative data on the 24 lines produced by conditioning treatments in variety 16 of Nicotiana rustica shows that for the mature plant characteristics exemplified by final plant height, the lines fall into three groups, large, intermediate and small. In this respect they are in broad qualitative agreement with the three genotrophs induced in flax, although it is not yet certain which of the three, if any, in $\mathcal{N}$. rustica corresponds to the original plastic genotroph. This classification, however, while of practical utility breaks down as more characters, particularly juvenile and developmental, are considered.
\end{abstract}

\section{IntRoduction}

Phenotypic differences produced by conditioning (Durrant, 1962) have been most thoroughly investigated in varieties of flax (Durrant, 1974), and Nicotiana rustica (Hill, 1965; Moore and Eglington, 1973). The conditioned lines or genotrophs in these two species share several common features (Perkins, Eglington and Jinks, 1971). At the same time, however, they differ in that the differences between the flax genotrophs appear to be more discontinuous than those between $\mathcal{N}$. rustica genotrophs. In flax the indicator character, plant size, divided the conditioned lines at an early stage into three classes, small, large and plastic, the latter being equivalent to the original unconditioned variety. Other differences both quantitative and qualitative were subsequently found to be associated with these three classes. In $\mathcal{N}$. rustica, on the other hand, no such grouping of lines proved possible. This was partly because the principal characters, flowering time and final height, differed in an uncorrelated way between the conditioned lines and partly because, from the outset, three independently conditioned lines had been maintained from each of the eight conditioning treatments and for some treatments these three lines were inconsistent in their performance. A considerable body of data has accumulated on the 24 conditioned lines since the original conditioning treatment of variety 16 in 1962 and this allows us to examine the validity of grouping the lines.

\section{THE GASE FOR THREE GENOTROPHS FOR FINAL HEIGHT}

The sources and dimensions of these data and the line means for final height and flowering time are summarised in tables 1,2 and 3. If initially we ignore all except the means for the eight conditioning treatments, averaged over seasons and triplicate lines of each treatment, then for final height (table 2) we appear to have a very simple picture. Two treatments 
$\mathrm{P}$ and PK have produced short phenotypes; two treatments NIL and NK have produced tall phenotypes and the remaining four treatments, intermediate phenotypes. At this level there is a direct correspondence with the three genotrophs of flax.

If we now extend our examination to the eight treatment means, averaged over triplicate lines, for each of the ten seasons individually we find that the members of the short and tall groups do not overlap in height in any season. A member of the intermediate group is shorter than one member of the short group in three seasons $\left(\mathrm{E}_{9}, \mathrm{E}_{10}\right.$ and $\left.\mathrm{E}_{13}\right)$ and another member of the intermediate group is as tall or taller than the members of the tall group in one season $\left(\mathbf{E}_{\mathbf{1 0}}\right)$ only. In the majority of seasons, therefore, we would

\section{TABLE 1}

Sources and dimensions of the data. The environment numbers correspond to those used by Hill and Perkins (1969)

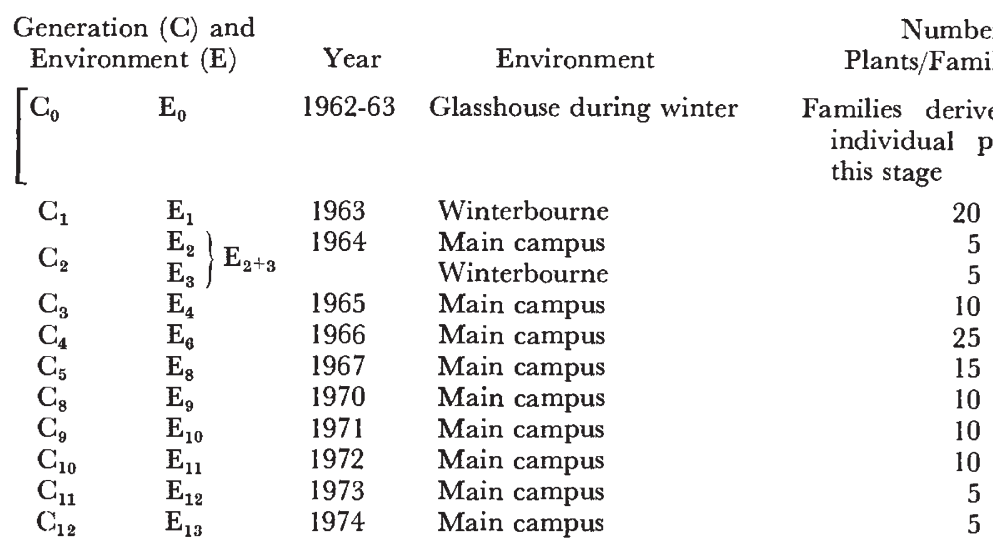

probably have arrived at the same qualitative groupings of the eight conditioning treatments. On the other hand, these comparisons reveal differences between treatments that have been placed in the same group. Of the four conditioning treatments assigned to the intermediate group, the one that received nitrogen $(\mathrm{N})$ produces the shortest plants in seven of the seasons as well as overall. The remaining three members of this group, in contrast, do not rank consistently relative to one another over the ten seasons and there is no reason to believe that they differ from one another. There is, however, a relatively consistent difference between the two treatments that fall into the short group, the $\mathrm{P}$ treatment being the shorter in eight of the ten seasons as well as on average over all seasons.

A two-way analysis of variance of the eight treatment means in each of the ten environments brings out these same points (table 4). In the first analysis involving all eight treatments there are highly significant differences overall between treatments and between environments relative to their interactions. When, however, the same analysis is carried out on the treatments after subdividing them into the three groups, there are differences between the two treatments of the short group and between the four treatments of the intermediate group but not between the two treatments of the 


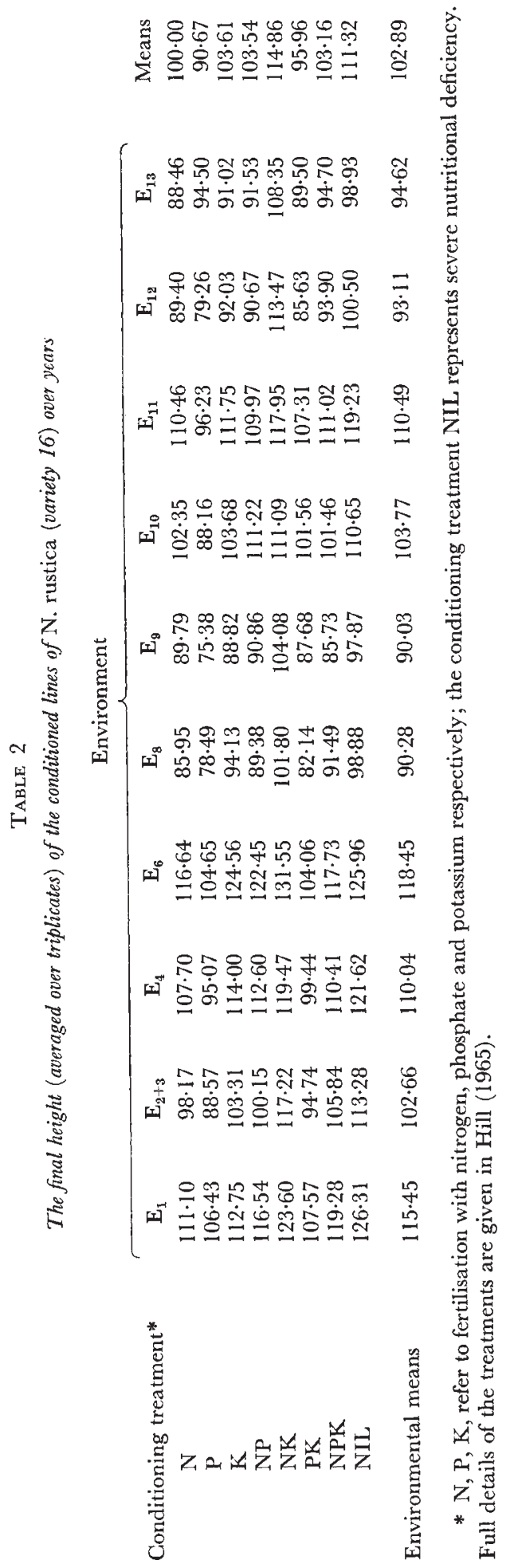




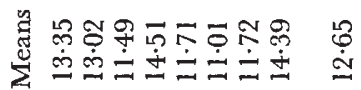
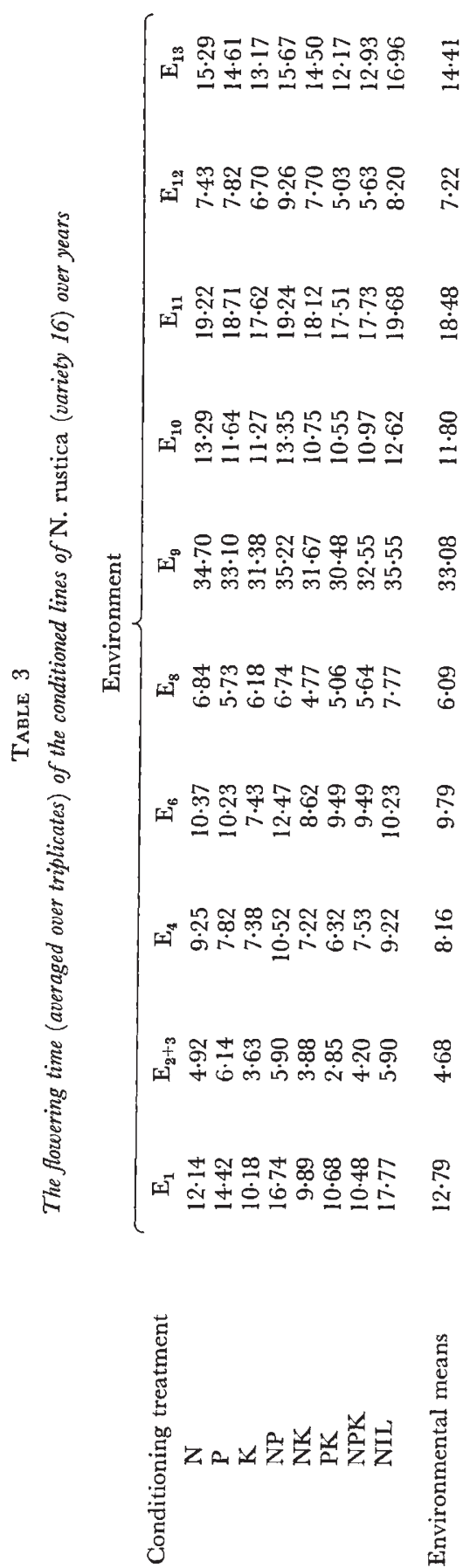


$$
\text { GONDITIONED LINES OF NICOTIANA }
$$


tall group. The significance of these differences is, however, much reduced and the differences within the intermediate group disappears completely on removing the $\mathrm{N}$ treatment which we previously noted was shorter than other members in most seasons. It could be argued, therefore, that the P, PK and $\mathrm{N}$ treatments have each produced a phenotypically distinct, short genotroph, the $\mathrm{K}, \mathrm{NP}$ and NPK treatments have all produced the same intermediate genotroph and the NK and NIL treatments the same large genotroph. An analysis of variance testing the grouping of the eight treatments into three short genotrophs, one intermediate genotroph and one tall genotroph is given in table 4. As expected the differences between the five groups account for all the significant differences among the eight treatments. All attempts to place them in a smaller number of groups led to significant differences between members of the same group.

We have now identified a homogeneous intermediate group and a homogeneous large group but each of the three short genotrophs appears to be unique. The reason can be found in the individual performances of the triplicate lines for each treatment. If we take the conditioning treatment, $\mathrm{P}$, which on average produces the shortest plants we find that two of the triplicate lines are identically short while the third is indistinguishable in height from the intermediate group. Similarly if we take the other conditioning treatments, $\mathrm{N}$ and $\mathrm{PK}$ which on average also produce short plants which are not as short as those from the $\mathbf{P}$ treatment, we find that in each case there is one line out of the three which is as short as the shorter of the P lines and two which are indistinguishable in height from the intermediate group. Thus the nine lines of the three conditioning treatments which produce the short genotrophs fall into two distinct groups, four being short and five being indistinguishable from the intermediate genotroph. As far as can be ascertained these two groups were present from the earliest generations following the conditioning treatments and are not the result of subsequent reversion from a short to an intermediate phenotype. Either, therefore, five of the nine plants exposed to these conditioning treatments failed to be conditioned or these treatments produced two alternative conditioning responses. The answer, of course, depends on whether the intermediate group is, as in flax, unconditioned like the original parental variety or a third conditioned state.

Although there are no significant heterogeneities among the six lines from the two conditioning treatments that produced the large genotroph, one of the three lines from the NIL treatment just overlaps with the intermediate group in its height and it has occupied this relative position consistently from the earliest records following the treatment. It is possible therefore, that this one line also did not respond to the conditioning treatment and is perhaps better regarded as a member of the intermediate group.

Variety 16 from which all the conditioned lines of $\mathcal{N}$. rustica were derived, has been compared with them on only one occasion in $1972\left(\mathrm{E}_{11}\right)$. Variety 16 had a final height of $100.46 \mathrm{~cm}$. This compares with a range of 86.72 to 124.28 among the conditioned lines with a mid-point of 105.50 and a mean of $110 \cdot 49$. The intermediate group had a mean of $112 \cdot 14$. On the limited evidence available, variety 16 while falling between the short and tall genotrophs, seems to be shorter than the intermediate genotroph. While this suggests that the intermediate genotroph is not the unconditioned variety 16 we cannot regard this single comparison as conclusive. 


\section{Flowering time and other Gharacters}

Although there are significant differences in flowering time among the conditioned lines comparable with those for final height there is no correlation between these two characters over the conditioned lines (Eglington, 1971 ; Eglington and Moore, 1973). Any grouping of the conditioning treatments based upon flowering time cannot, therefore, coincide with the grouping we obtained for final height. Nevertheless on the basis of the flowering times of the eight conditioning treatments after averaging over triplicate lines and all ten environments (table 3) we can recognise three groups. There is an early flowering group that contains all conditioning treatments that included potassium (K, NK, PK and NPK), an intermediate group containing the two conditioning treatments $\mathrm{N}$ and $\mathrm{P}$ and a late flowering group containing the remaining treatments NP and NIL. Hence, of the nine possible combinations of tall, intermediate and short for final height and early, intermediate and late for flowering time seven are represented among the eight conditioning treatments. Only two, K and NPK, can still be grouped, both being intermediate in height and early flowering.

For flowering time, as for final height, we can, therefore, recognise three distinct phenotypic groups resulting from the eight conditioning treatments but with the exception of K and NPK each conditioning treatment produces its own unique phenotype when both characters are considered simultaneously. In all, up to 23 characters have been recorded for the conditioned lines in any one experiment and seven or more of these have been recorded on at least three occasions (Eglington, 1971; Moore, 1974). These characters have fallen into four independent classes on a principal component analysis. Final height was the highest loading factor in the first of these components which included all the mature plant characteristics. Not surprisingly all the characters led to the same grouping of the conditioned lines as those described earlier for final height.

The second principal component consisted mainly of floral and capsule characters. For these characters the NP conditioned line (intermediate height, late flowering) was invariably the largest genotroph and the $P$ conditioned line (short and intermediate in flowering) the smallest. This component, unlike the first, however, does not allow any clear-cut grouping of the remaining lines. The third component which consists mainly of the number of vegetative nodes and the maximum breadth of the leaf lamina also allows no grouping. The fourth component, however, because it consists mainly of flowering time leads to the grouping already described.

Each component leads to its own independent ranking of the conditioned lines and overall, therefore, each conditioning treatment has led to a unique phenotype. Furthermore, there is no pair of conditioned lines which are opposite extremes for all characters. On the other hand, the first two components are responsible for most of the variation and by far the largest number of characters are assignable to them. These two components are also consistent to the extent that the $\mathrm{P}$ conditioning treatment produces the smallest genotroph on both of them. Although no single classification will hold over all stages of development, for practical purposes, a classification based upon final height would be the most useful because it applies to several characters, particularly the mature characters, and, as we have already seen, it is consistent over seasons. 


\section{Conclusions}

If we confine our attention to the differences in size displayed by mature plants the effect of the conditioning treatments on variety 16 of $\mathcal{N}$. rustica has been to produce large, intermediate and small genotrophs. In this respect, therefore, the effect of conditioning in $\mathcal{N}$. rustica is qualitatively the same as in flax. In $\mathcal{N}$. rustica, however, it has still not yet been established whether one of these three genotrophs is the unchanged, conditionable variety 16 although limited comparisons suggest that the original variety falls between the intermediate and small genotrophs for most characters. As more characters have been scored on the conditioned lines of flax and $\mathcal{N}$. rustica however, it has become increasingly clear that while the classification into these three types is satisfactory for most practical purposes and is particularly useful in planning experimental comparisons, it is an oversimplification. Indeed if all the characters which have been measured on the 24 conditioned lines of $\mathcal{N}$. rustica maintained at Birmingham are taken into account almost every line has a unique phenotype.

\section{REFERENCES}

DURRANT, A. 1962. The environmental induction of heritable changes in Linum. Heredity, $17,27-61$.

DURRANT, A. 1974. The association of induced changes in flax. Heredity, 32, 133-144.

EGLINGTON, E. G. 1971. Genotype-environment interactions in plants. Ph.D. thesis, University of Birmingham.

EGLINGTON, E. G., AND MOORE .c. A. 1973. The nature of the inheritance of permanently induced changes in Nicotiana rustica. II. Analysis of segregation in the $\mathrm{F}_{4}$ and $\mathrm{F}_{5}$ generations of selected crosses. Heredity, 30, 387-395.

HILL, J. 1965. Environmental induction of heritable changes in Nicotiana rustica. Nature, 207, 732-734.

HILL, J., AND PERKINS, J. M. 1969. The environmental induction of heritable changes in Nicotiana rustica. Effects of Genotype-environment interactions. Genetics, 61, 661-675.

MOORE, C. A., AND EGLINGTON, E. G. 1973. The nature of the inheritance of permanently induced changes in Nicotiana rustica. III. $\mathrm{F}_{5}$ generation for five characters. Heredity, 31, $112-118$.

MOORE, C. A. 1974. Conditioned lines of Nicotiana rustica. Ph.D. thesis, University of Birmingham.

PERKINS, J. M., EGLINGTON, E. G., AND JINKS, J. L. 1971. The nature of the inheritance of permanently induced changes in Nicotiana rustica. Heredity, 27, 441-457. 